\title{
PRECONDITIONED ITERATIVE METHODS FOR SOLVING TOEPLITZ-PLUS-HANKEL SYSTEMS*
}

\author{
TA-KANG $\mathrm{KU}^{\dagger}$ AND C.-C. JAY KUO ${ }^{\dagger}$
}

\begin{abstract}
The use of preconditioned iterative methods to solve a system of equations with a Toeplitz-plus-Hankel coefficient matrix is studied. A new preconditioner suitable for Toeplitzplus-Hankel matrices is proposed, and the spectral properties of preconditioned rational Toeplitzplus-Hankel matrices are examined. It is shown that the eigenvalues of the preconditioned matrix are clustered around unity, except for a finite number of outliers, depending on the orders of the rational generating functions, and the clustering radius is proportional to the magnitude of the last elements in Toeplitz and Hankel matrices. With the spectral regularities, an $N \times N$ rational Toeplitzplus-Hankel system can be solved by preconditioned iterative methods with $O(N \log N)$ operations. Numerical experiments are given to demonstrate the efficiency of the proposed preconditioner.
\end{abstract} tion

Key words. Toeplitz-plus-Hankel, preconditioned iterative method, rational generating func-

AMS subject classifications. $65 \mathrm{~F} 10,65 \mathrm{~F} 15$

1. Introduction. The systems of linear equations with Toeplitz, Hankel, and Toeplitz-plus-Hankel coefficient matrices arise in many signal processing applications. For example, the inverse scattering problem can be formulated as Toeplitz, Hankel, and Toeplitz-plus-Hankel systems of equations, which were done by Krein [20], Agranovich and Marchenko [1], and Gelfand and Levitan [14], respectively. (For more recent work, we refer to [3], [4].) By exploiting the special structures of Toeplitz or Hankel matrices, an $N \times N$ system of equations can be solved by fast direct methods based on the Levinson or Schur algorithm with $O\left(N^{2}\right)$ operations [11], [12], [13], [21]. Direct algorithms for inverting $N \times N$ Toeplitz-plus-Hankel matrices with $O\left(N^{2}\right)$ complexity have also been derived [16], [17], [27]. Although the computational complexity of these fast algorithms is lower than that of the Gaussian elimination with pivoting, i.e., $O\left(N^{3}\right)$, their stability is not guaranteed when applied to indefinite or nonsymmetric matrices [5], [10]. In this paper, we propose to use preconditioned iterative methods to solve Toeplitz-plus-Hankel systems, which have a low computational complexity and a stable convergence performance.

Toeplitz preconditioners in circulant or skew-circulant matrix form have been proposed and analyzed by many researchers [7], [9], [19], [22], [30], [32]. It was shown by Chan and Strang [8] that, for a large class of symmetric Toeplitz matrices, the eigenvalues of preconditioned matrices are clustered around unity and the preconditioned iterative method converges at a superlinear rate. For Toeplitz matrices generated by rational functions, an even stronger convergence result was obtained by Trefethen [31] and the authors [24], [25]. That is, the preconditioned iterative method converges in a finite number of iterations independent of the problem size $N$. Consequently, a rational Toeplitz system can be solved with $O(N \log N)$ operations. In addition to low computational complexity, preconditioned iterative methods demonstrate a very stable convergence behavior. Since a Hankel system can be transformed to a Toeplitz system by reversing the order of the linear equations, the same results also hold for

* Received by the editors June 10, 1991; accepted for publication (in revised form) July 8, 1992. This work was supported by the University of Southern California Faculty Research and Innovation Fund and by National Science Foundation Research Initiation Award ASC-9009323.

$\dagger$ Signal and Image Processing Institute and Department of Electrical Engineering Systems, University of Southern California, Los Angeles, California 90089-2564. 
Hankel systems.

The inverse scattering problem is often formulated as two waves propagating in opposite directions. The discrete version of the formulation can be naturally expressed as an $N \times N$ Toeplitz-plus-Hankel system $A \mathbf{x}=\mathbf{b}$, where $A$ is the sum of a Toeplitz matrix $T$ and a Hankel matrix $H$ with elements $T_{i, j}=t_{i-j}$ and $H_{i, j}=h_{N+1-(i+j)}$ [2], [3], [4], [6], [26]. The idea of constructing a Toeplitz-plus-Hankel preconditioner can be simply stated as follows. Let $J$ be an $N \times N$ matrix which has ones along the secondary diagonal and zeros elsewhere (i.e., $J_{i, j}=1$ if $i+j=N+1$ and $J_{i, j}=0$ if $i+j \neq N+1)$. We can easily verify that the product of $J$ and $H$ gives a Toeplitz matrix $T_{H}=J H$ with elements $\left[T_{H}\right]_{i, j}=h_{i-j}$, and that the Toeplitz-plus-Hankel matrix can be expressed as $A=T+H=T+J T_{H}$. Now, given preconditioners $K_{T}$ and $K_{H}$ for Toeplitz matrices $T$ and $T_{H}$, we propose to use $P=K_{T}+J K_{H}$ as a preconditioner for matrix $A$.

To solve the Toeplitz-plus-Hankel system $A \mathbf{x}=\mathbf{b}$ with preconditioner $P$, two major computations required at each iteration are the matrix-vector products $A \mathbf{v}$ and $P^{-1} \mathbf{v}$ with an arbitrary vector $\mathbf{v}$. The operation $A \mathbf{v}$ can be performed effectively via fast Fourier transform (FFT) with $O(N \log N)$ operations, since both $T \mathbf{v}$ and $H \mathbf{v}$ can be embedded in a $2 N \times 2 N$ circulant matrix-vector product. To implement the preconditioning step $P \mathbf{z}=\mathbf{v}$, we relate it to an equivalent $N \times N$ circulant system which can be inverted via FFT with $O(N \log N)$ operations. Consequently, the computational complexity for each iteration is $O(N \log N)$.

In the context of inverse scattering, the generating sequences of Toeplitz and Hankel matrices can be selected with great flexibility. Thus, we focus on the case that the sequences $\left\{t_{n}\right\}$ and $\left\{h_{n}\right\}$ are generated, respectively, by rational functions of orders $\left(\alpha_{T}, \beta_{T}, \gamma_{T}, \delta_{T}\right)$ and $\left(\alpha_{H}, \beta_{H}, \gamma_{H}, \delta_{H}\right)$ (see the definition in $\left.\S 3.1\right)$ and study the spectral properties of preconditioned matrices. The eigenvalues of $P^{-1} A$ are classified into two classes, i.e., the outliers and the clustered eigenvalues. Then the preconditioned matrix has the following two spectral properties: (1) The number of outliers is bounded by a constant which depends on the orders of the rational generating functions; and (2) the clustered eigenvalues are confined in a disk centered at unity with radius $\epsilon$ proportional to $O\left(\left|t_{N}\right|+\left|t_{-N}\right|+\left|h_{N}\right|+\left|h_{-N}\right|\right)$. With the above spectral properties, various preconditioned terative methods, including CGN (the Conjugate Gradient iteration applied to the Normal equations) [18], GMRES (the Generalized Minimal Residual) [28], and CGS (the Conjugate Gradient Square) [29], can be effectively applied. It turns out that a rational Toeplitz-plus-Hankel system can be solved in a finite number of iterations independent of the problem size $N$ so that the total operations required are $O(N \log N)$. Besides, preconditioned iterative methods are highly parallelizable due to the parallelism provided by FFT. The time complexity can be reduced to $O(\log N)$ if $O(N)$ processors are used.

I his paper is organized as follows. We discuss the construction and the computational complexity of preconditioner $P$ for Toeplitz-plus-Hankel matrices in $\S 2$. The spectral properties of the preconditioner and the preconditioned rational Toeplitzplus-Hankel matrix are examined in $\S 3$. Numerical experiments are given in $\S 4$ to illustrate our theoretical study.

\section{The Toeplitz-plus-Hankel preconditioner.}

2.1. Construction of the preconditioner. Consider the $N \times N$ Toeplitz-plusHankel system

$$
A \mathbf{x}=\mathbf{b}, \quad A=T+H
$$


where $T$ and $H$ are given Toeplitz and Hankel matrices with elements $T_{i, j}=t_{i-j}$ and $H_{i, j}=h_{N+1-(i+j)}$. With the special structures of Toeplitz and Hankel matrices, a Hankel matrix $H$ can be transformed to a Toeplitz matrix by premultiplying (or postmultiplying) it with

$$
J=\left[\begin{array}{lllll}
0 & 0 & \cdot & 0 & 1 \\
0 & \cdot & 0 & 1 & 0 \\
\cdot & \cdot & \cdot & \cdot & \cdot \\
0 & 1 & 0 & . & 0 \\
1 & 0 & \cdot & 0 & 0
\end{array}\right]
$$

which is known as the time-reversal operator. It is clear that $T_{H}=J H$ is a Toeplitz matrix with elements $\left[T_{H}\right]_{i, j}=h_{i-j}$ and that $J T_{H}=J^{2} H=H$. Thus, (2.1) can also be written as

$$
\left(T+J T_{H}\right) \mathbf{x}=\mathbf{b}
$$

where $T$ and $T_{H}$ are $N \times N$ Toeplitz matrices generated by the sequences $\left\{t_{n}\right\}$ and $\left\{h_{n}\right\}$, respectively.

The procedure to construct a circulant preconditioner for Toeplitz matrix

$$
T=\left[\begin{array}{ccccc}
t_{0} & t_{-1} & \cdot & t_{-(N-2)} & t_{-(N-1)} \\
t_{1} & t_{0} & t_{-1} & \cdot & t_{-(N-2)} \\
\cdot & t_{1} & t_{0} & \cdot & \cdot \\
t_{N-2} & \cdot & \cdot & \cdot & t_{-1} \\
t_{N-1} & t_{N-2} & \cdot & t_{1} & t_{0}
\end{array}\right]
$$

is summarized as follows [22]. We are motivated by the observation that we can solve the $2 N \times 2 N$ circulant system effectively,

$$
R_{T}\left[\begin{array}{l}
\mathbf{x} \\
\mathbf{x}
\end{array}\right]=\left[\begin{array}{l}
\mathbf{b} \\
\mathbf{b}
\end{array}\right], \quad R_{T}=\left[\begin{array}{cc}
T & \triangle T \\
\triangle T & T
\end{array}\right]
$$

where $\Delta T$ is determined by the elements of $T$ to make $R_{T}$ circulant, i.e.,

$$
\Delta T=\left[\begin{array}{ccccc}
0 & t_{N-1} & \cdot & t_{2} & t_{1} \\
t_{-(N-1)} & 0 & t_{N-1} & \cdot & t_{2} \\
\cdot & t_{-(N-1)} & 0 & \cdot & \cdot \\
t_{-2} & \cdot & \cdot & \cdot & t_{N-1} \\
t_{-1} & t_{-2} & \cdot & t_{-(N-1)} & 0
\end{array}\right]
$$

Since (2.3) is equivalent to

$$
K_{T} \mathbf{x}=\mathbf{b}, \quad K_{T}=T+\triangle T
$$

we choose the circulant matrix $K_{T}$ as a preconditioner for $T$. Similarly, we have the circulant preconditioner $K_{H}=T_{H}+\Delta T_{H}$ for Toeplitz matrix $T_{H}$. Then, with respect to (2.2), we propose to use

$$
P=K_{T}+J K_{H}
$$

as a preconditioner for the Toeplitz-plus-Hankel matrix $A$. 
2.2. Computational complexity of the preconditioner. To solve the Toeplitz-plus-Hankel system $A \mathbf{x}=\mathbf{b}$ with preconditioner $P$, two major computations required at each iteration are the matrix-vector products $A \mathbf{v}$ and $P^{-1} \mathbf{v}$ with an arbitrary vector $\mathbf{v}$. We show below that they both can be achieved with $O(N \log N)$ operations. Since other operations involved are vector additions or inner products whose complexity is proportional to $O(N)$, the total computational complexity per iteration is $O(N \log N)$.

The $N \times N$ Toeplitz matrix-vector product $T \mathbf{v}$ can be embedded in the $2 N \times 2 N$ circulant matrix-vector product

$$
\left[\begin{array}{cc}
T & \Delta T \\
\triangle T & T
\end{array}\right]\left[\begin{array}{l}
\mathbf{v} \\
\mathbf{0}
\end{array}\right]=\left[\begin{array}{c}
T \mathbf{v} \\
\triangle T \mathbf{v}
\end{array}\right]
$$

so that it can be effectively computed via FFT with $O(N \log N)$ operations. Premultiplying $J$ to a vector $\mathbf{v}$ corresponds to the reverse of the order of the elements in $\mathbf{v}$. Thus, the Toeplitz-plus-Hankel matrix-vector product

$$
A \mathbf{v}=T \mathbf{v}+J T_{H} \mathbf{v}
$$

can also be achieved with $O(N \log N)$ operations.

With the equality $J^{2}=I$ and (2.5), we have

$$
P \mathbf{z}=K_{T} \mathbf{z}+J K_{H} J J \mathbf{z}=\mathbf{v},
$$

which is equivalent to

$$
J P \mathbf{z}=J K_{T} J J \mathbf{z}+K_{H} \mathbf{z}=J \mathbf{v} .
$$

Since $K_{T}$ and $K_{H}$ are circulant, $J K_{T} J=K_{T}^{T}, J K_{H} J=K_{H}^{T}$ and $K_{T}$, and $K_{H}$ commute. By multiplying (2.6) with $K_{T}^{T}$ and (2.7) with $K_{H}^{T}$, we can write the difference between the two resulting equations as

$$
\left(K_{T}^{T} K_{T}-K_{H}^{T} K_{H}\right) \mathbf{z}=K_{T}^{T} \mathbf{v}-K_{H}^{T} J \mathbf{v} .
$$

Thus, the solution of $\mathbf{z}=P^{-1} \mathbf{v}$ can also be determined from (2.8). It is easy to verify that $K_{T}^{T} K_{T}-K_{H}^{T} K_{H}$ is circulant and can be diagonalized with Fourier functions. Consequently, $P^{-1} \mathbf{v}$ can be solved effectively via FFT with $O(N \log N)$ complexity. Note also that the Fourier coefficients $\hat{v}_{k}$ and $\hat{w}_{k}$ of the vectors $\mathbf{v}$ and $\mathbf{w}=J \mathbf{v}$ are related via

$$
\hat{w}_{k}=\hat{v}_{-k \bmod N}, \quad 0 \leq k \leq N-1,
$$

which means that only a FFT and an inverse FFT are needed in solving (2.8), given the eigenvalues of $K_{T}$ and $K_{H}$.

In conclusion, the total computational complexity inside each iteration of the preconditioned iterative methods is only $O(N \log N)$. Besides, the preconditioned iterative methods are highly parallelizable due to the parallelism provided by FFT, and the time complexity can be reduced to $O(\log N)$ if $O(N)$ processors are used.

3. Analysis of the preconditioner. We assume that Toeplitz matrix $T$ and Hankel matrix $H$ are generated by real sequences $\left\{t_{n}\right\}$ and $\left\{h_{n}\right\}$ satisfying

$$
\sum_{-\infty}^{\infty}\left|t_{n}\right| \leq B_{T}<\infty, \quad \sum_{-\infty}^{\infty}\left|h_{n}\right| \leq B_{H}<\infty .
$$


The circulant matrices $K_{T}, K_{T}^{T}, K_{H}$, and $K_{H}^{T}$ share the same Fourier functions as their eigenvectors with eigenvalues

$$
\begin{aligned}
& \lambda_{k}\left(K_{T}\right)=\lambda_{k}^{*}\left(K_{T}^{T}\right)=\sum_{n=-(N-1)}^{N-1} t_{n} e^{-j(2 \pi k n / N)} \\
& \lambda_{k}\left(K_{H}\right)=\lambda_{k}^{*}\left(K_{H}^{T}\right)=\sum_{n=-(N-1)}^{N-1} h_{n} e^{-j(2 \pi k n / N)},
\end{aligned}
$$

where $k=0,1, \ldots, N-1$, and $\lambda_{k}^{*}$ denotes the complex conjugate of $\lambda_{k}$. We also assume that

$$
\left.|| \lambda_{k}\left(K_{T}\right)\right|^{2}-\left|\lambda_{k}\left(K_{H}\right)\right|^{2} \mid \geq \mu>0, \quad 0 \leq k \leq N-1
$$

where $\mu$ is a constant independent of $N$. We will show in Theorem 1 that the above condition implies the invertibility of the preconditioner $P$.

The generating functions of $T$ and $T_{H}$ are defined as

$$
T(z)=\sum_{n=-\infty}^{\infty} t_{n} z^{-n}, \quad H(z)=\sum_{n=-\infty}^{\infty} h_{n} z^{-n} .
$$

We focus on the case

$$
T(z)=\frac{A_{T}\left(z^{-1}\right)}{B_{T}\left(z^{-1}\right)}+\frac{C_{T}(z)}{D_{T}(z)}, \quad H(z)=\frac{A_{H}\left(z^{-1}\right)}{B_{H}\left(z^{-1}\right)}+\frac{C_{H}(z)}{D_{H}(z)},
$$

where $T(z)$ is a rational function of order $\left(\alpha_{T}, \beta_{T}, \gamma_{T}, \delta_{T}\right)$, i.e.,

$$
\begin{array}{ll}
A_{T}\left(z^{-1}\right)=\sum_{i=0}^{\alpha_{T}} a_{T, i} z^{-i}, & C_{T}(z)=\sum_{i=0}^{\gamma_{T}} c_{T, i} z^{i}, \\
B_{T}\left(z^{-1}\right)=\sum_{i=0}^{\beta_{T}} b_{T, i} z^{-i}, & D_{T}(z)=\sum_{i=0}^{\delta_{T}} d_{T, i} z^{i},
\end{array}
$$

with $a_{T, \alpha_{T}} b_{T, \beta_{T}} c_{T, \gamma_{T}} d_{T, \delta_{T}} \neq 0, b_{T, 0}=1, d_{T, 0}=1$, and polynomials $A_{T}\left(z^{-1}\right)$ and $B_{T}\left(z^{-1}\right)$ (or $C_{T}\left(z^{-1}\right)$ and $D_{T}\left(z^{-1}\right)$ ) have no common factors. Similarly, $H(z)$ is a rational function of order $\left(\alpha_{H}, \beta_{H}, \gamma_{H}, \delta_{H}\right)$. For convenience, we let

$$
\begin{array}{ll}
r_{T}=\max \left(\alpha_{T}, \beta_{T}\right), & s_{T}=\max \left(\gamma_{T}, \delta_{T}\right), \\
r_{H}=\max \left(\alpha_{H}, \beta_{H}\right), & s_{H}=\max \left(\gamma_{H}, \delta_{H}\right) .
\end{array}
$$

Note that (3.1) implies that all zeros of $B_{T}\left(z^{-1}\right)$ and $B_{H}\left(z^{-1}\right)\left(D_{T}(z)\right.$ and $\left.D_{H}(z)\right)$ are inside (outside) the unit circle. By applying the isomorphism between the ring of the power series and the ring of semi-infinite lower (or upper) triangular Toeplitz matrices, with respect to $T(z)$ and $H(z)$ given in (3.3), we have

$$
T=L_{A, T} L_{B, T}^{-1}+U_{C, T} U_{D, T}^{-1}, \quad T_{H}=L_{A, H} L_{B, H}^{-1}+U_{C, H} U_{D, H}^{-1},
$$

where $L_{A, T}$ and $L_{B, T}\left(U_{C, T}\right.$ and $\left.D_{C, T}\right)$ are $N \times N$ lower (upper) triangular Toeplitz matrices generated by $A_{T}\left(z^{-1}\right)$ and $B_{T}\left(z^{-1}\right)\left(C_{T}(z)\right.$ and $\left.D_{T}(z)\right)$, respectively. The $L_{A, H}, L_{B, H}, U_{C, H}$, and $U_{D, H}$ are similarly defined with respect to $H(z)$. 
3.1. Invertibility of the preconditioner. With condition (3.2), in the following theorem we prove that $P^{-1}$ exists.

THEOREM 1. Let $T$ and $H$ be $N \times N$ Toeplitz and Hankel matrices satisfying (3.2). Then, $\left\|P^{-1}\right\|_{2}$ is bounded for any given $N$.

Proof. Recall from (2.8) that $P \mathbf{z}=\mathbf{v}$ is equivalent to

$$
\left(K_{T}^{T} K_{T}-K_{H}^{T} K_{H}\right) \mathbf{z}=\left(K_{T}^{T}-K_{H}^{T} J\right) \mathbf{v} .
$$

For any given $N,\left\|K_{T}^{T}\right\|_{1}$ and $\left\|K_{T}^{T}\right\|_{\infty}$ are both bounded. As a consequence, $\left\|K_{T}^{T}\right\|_{2}$ is also bounded. Similar results hold for $K_{H}^{T}$. Thus, the right-hand side of (3.6) is bounded. With condition (3.2), the magnitude of any eigenvalue of $K_{T}^{T} K_{T}-K_{H}^{T} K_{H}$ is also bounded by

$$
\left|\lambda_{k}\left(K_{T}^{T} K_{T}-K_{H}^{T} K_{H}\right)\right|=\left.|| \lambda_{k}\left(K_{T}\right)\right|^{2}-\left|\lambda_{k}\left(K_{H}\right)\right|^{2} \mid \geq \mu>0 .
$$

Therefore, $\left\|P^{-1}\right\|_{2}$ is bounded and the preconditioner $P$ is invertible.

3.2. The number of outliers of $P^{-1} A$. Since

$$
\lambda\left(P^{-1} A\right)=1-\lambda\left(P^{-1} \triangle A\right)
$$

the eigenvalues of $P^{-1} A$ clustered around one correspond to those of $P^{-1} \triangle A$ clustered around zero. According to the definitions of $K_{T}$ and $K_{H}$, the difference matrix $\triangle A=P-A$ can be written as

$$
\triangle A=\triangle T+J \triangle T_{H}
$$

Based on the structures of $\triangle T$ and $\triangle T_{H}$, we will decompose the $\triangle A$ into the sum of a low rank matrix $\triangle F$ and a perturbation matrix, in which the rank of $\triangle F$ does not change with $N$ and all matrix norms of the perturbation matrix converge to zero for asymptotically large $N$. Then, we define the outliers of $P^{-1} \triangle A$ as the eigenvalues of $P^{-1} \triangle A$ corresponding to the nonzero eigenvalues of $P^{-1} \triangle F$ with perturbation. Since there is a one-to-one correspondence between the eigenvalues of $P^{-1} \triangle A$ and $P^{-1} A$, the outliers of $P^{-1} A$ can also be defined. A direct consequence of this definition is that the outliers of $P^{-1} A$ do not converge to unity for asymptotically large $N$. Besides, the number of outliers of $P^{-1} A$ is fixed and equivalent to the rank of $\triangle F$. The rank of $\triangle F$ will be examined in this subsection so that the number of the outliers is determined. The perturbation theory of eigenvalues will then be used in $\S 3.3$ to study the clustering radius of the clustered eigenvalues.

The analysis for the number of outliers proceeds as follows.

Step 1. Construct a low rank matrix $\triangle F$ based on the recursion in $t_{n}$ and $h_{n}$ described in Lemma 1.

Step 2. Show that $\triangle F$ is asymptotically equivalent to $\triangle A$ (Lemma 2).

Step 3. Establish an upper bound for the rank of $\triangle F$, which is equal to the number of outliers of $P^{-1} A$ (Theorem 2).

LEMMA 1. The sequences $t_{n}$ and $h_{n}$ generated by (3.3) that satisfy condition (3.1) follow the recursions

$$
\begin{array}{ll}
t_{n+1}=-\left(b_{T, 1} t_{n}+b_{T, 2} t_{n-1}+\cdots+b_{T, \beta_{T}} t_{n-\beta_{T}+1}\right), & n \geq r_{T}, \\
t_{n-1}=-\left(d_{T, 1} t_{n}+d_{T, 2} t_{n+1}+\cdots+d_{T, \delta_{T}} t_{n+\delta_{T}-1}\right), & n \leq-s_{T}, \\
h_{n+1}=-\left(b_{H, 1} h_{n}+b_{H, 2} h_{n-1}+\cdots+b_{H, \beta_{H}} h_{n-\beta_{H}+1}\right), & n \geq r_{H}, \\
h_{n-1}=-\left(d_{H, 1} h_{n}+d_{H, 2} h_{n+1}+\cdots+d_{H, \delta} h_{n+\delta_{H}-1}\right), & n \leq-s_{H},
\end{array}
$$


where $b_{T, i}, d_{T, i}, b_{H, i}$, and $d_{H, i}$ are given in $(3.3 \mathrm{~b})$ and $r_{T}, s_{T}, r_{H}$, and $s_{H}$ are defined in (3.4).

Proof. The proof is similar to the proof of Lemma 1 in [24].

Based on the recursion (3.8), we construct two low rank Toeplitz matrices $\triangle F_{T}$ and $\triangle H_{T}$ as

$$
\triangle F_{T}=F_{T, 1}+F_{T, 2}, \quad \text { and } \quad \Delta F_{H}=F_{H, 1}+F_{H, 2},
$$

where

$$
\begin{gathered}
F_{T, 1}=\left[\begin{array}{ccccc}
t_{N} & t_{N-1} & \cdot & t_{2} & t_{1} \\
t_{N+1} & t_{N} & t_{N-1} & \cdot & t_{2} \\
\cdot & t_{N+1} & t_{N} & \cdot & \cdot \\
t_{2 N-2} & \cdot & \cdot & \cdot & t_{N-1} \\
t_{2 N-1} & t_{2 N-2} & \cdot & t_{N+1} & t_{N}
\end{array}\right], \\
F_{T, 2}=\left[\begin{array}{ccccc}
t_{-N} & t_{-(N+1)} & \cdot & t_{-(2 N-2)} & t_{-(2 N-1)} \\
t_{-(N-1)} & t_{-N} & t_{-(N+1)} & \cdot & t_{-(2 N-2)} \\
\cdot & t_{-(N-1)} & t_{-N} & \cdot & \cdot \\
t_{-2} & \cdot & \cdot & \cdot & t_{-(N+1)} \\
t_{-1} & t_{-2} & \cdot & t_{-(N-1)} & t_{-N}
\end{array}\right],
\end{gathered}
$$

and $F_{H, 1}$ and $F_{H, 2}$ are similarly constructed by changing elements of $F_{T, 1}$ and $F_{T, 2}$ from $t_{n}$ to $h_{n}$. The $\Delta F_{T}$ and $\Delta F_{H}$ are asymptotically equivalent to $\Delta T$ and $\Delta T_{H}$, respectively, and we have the following lemma.

LEMMA 2. Let $T$ and $H$ be $N \times N$ Toeplitz and Hankel matrices generated by $T(z)$ and $H(z)$ in (3.3) with the corresponding generating sequences satisfying (3.1) and (3.2). Then,

$$
\|\triangle A-\triangle F\|_{2} \leq O\left(\left|t_{N}\right|+\left|t_{-N}\right|+\left|h_{N}\right|+\left|h_{-N}\right|\right),
$$

where $\triangle A$ is defined in (3.7) and

$$
\triangle F=\triangle F_{T}+J \triangle F_{H}
$$

with $\triangle F_{T}$ and $\triangle F_{H}$ given by (3.9). Consequently, $\triangle A$ is asymptotically equivalent to $\triangle F$.

Proof. The proof is similar to the proof of Lemma 2 in [25].

With (3.8), we can easily determine upper bounds for the ranks of $\triangle F_{T}$ and $\triangle F_{H}$, i.e.,

$$
\operatorname{rank}\left(\Delta F_{T}\right) \leq r_{T}+s_{T} \quad \text { and } \quad \operatorname{rank}\left(\Delta F_{H}\right) \leq r_{H}+s_{H}
$$

Since both $J$ and $P$ are full rank matrices, the rank of $P^{-1} \triangle F$ (or equivalently the number of outliers of $P^{-1} A$ ) is bounded above by

$$
\tilde{\eta}=r_{T}+s_{T}+r_{H}+s_{H} .
$$

However, the bound $\tilde{\eta}$ is not tight. A tighter bound is available according to the following theorem. 
TheOREM 2. Let $T$ and $H$ be $N \times N$ Toeplitz and Hankel matrices generated by $T(z)$ and $H(z)$ in (3.3) with the corresponding generating sequences satisfying (3.1) and (3.2). The number of outliers of $P^{-1} A$ is bounded by

$$
\eta=\max \left(r_{T}+\beta_{H}, r_{H}+\beta_{T}\right)+\max \left(s_{T}+\delta_{H}, s_{H}+\delta_{H}\right)-\eta_{c}
$$

where $\eta_{c}$ is the number of common roots in $B_{T}(z) D_{T}(z)$ and $B_{H}(z) D_{H}(z)$.

Proof. We first focus on the case that all roots of $B_{T}(z), D_{T}(z), B_{H}(z)$, and $D_{H}(z)$ are simple. By applying the partial fractional expansion to $A_{T}\left(z^{-1}\right) / B_{T}\left(z^{-1}\right)$ and $C_{T}(z) / D_{T}(z)$ and determining the corresponding Toeplitz matrix for each term, we obtain a decomposition for $T$, i.e.,

$$
T=T_{1,0}+\sum_{i=1}^{\beta_{T}} T_{1, i}+T_{2,0}+\sum_{i=1}^{\delta_{T}} T_{2, i}
$$

In the above expression, $T_{1, i}$ and $T_{2, i}$ are, respectively, lower and upper triangular Toeplitz matrices. If $\alpha_{T}-\beta_{T} \geq 0, T_{1,0}$ has a lower bandwidth $\alpha_{T}-\beta_{T}$. Otherwise, it is equal to zero. The $T_{1, i}, i \neq 0$, corresponds to the Toeplitz matrix generated by a root of $B_{T}\left(z^{-1}\right)$. The $T_{2, i}, 0 \leq i \leq \delta_{T}$, is similarly defined.

We construct $F_{T, 1, i}$ and $F_{T, 2, i}$ for $T_{1, i}$ and $T_{2, i}$ based on (3.9). Since the construction is linear, we have

$$
\triangle F_{T}=F_{T, 1,0}+\sum_{i=1}^{\beta_{T}} F_{T, 1, i}+F_{T, 2,0}+\sum_{i=1}^{\delta_{T}} F_{T, 2, i} .
$$

It is easy to verify that the elements of $F_{T, 1,0}$ and $F_{T, 2,0}$ are zeros except the northeast $\max \left(\alpha_{T}-\beta_{T}, 0\right)$ and the southwest $\max \left(\gamma_{T}-\delta_{T}, 0\right)$ diagonals, respectively. All remaining terms in $\triangle F_{T}$ are rank one matrices. The $\triangle F_{H}$ can be similarly decomposed as

$$
\Delta F_{H}=F_{H, 1,0}+\sum_{i=1}^{\beta_{H}} F_{H, 1, i}+F_{H, 2,0}+\sum_{i=1}^{\delta_{H}} F_{H, 2, i}
$$

where the elements of $F_{H, 1,0}$ and $F_{H, 2,0}$ are zeros except the northeast $\max \left(\alpha_{H}-\beta_{H}, 0\right)$ and the southwest $\max \left(\gamma_{H}-\delta_{H}, 0\right)$ diagonals, respectively, and all other terms are rank one matrices.

Let us examine the rank of $\triangle F=\Delta F_{T}+J \Delta F_{H}$. The ranks of $F_{T, 1,0}+J F_{H, 1,0}$ and $F_{T, 2,0}+J F_{H, 2,0}$ are clearly bounded by $\max \left(\alpha_{T}-\beta_{T}, \alpha_{H}-\beta_{H}, 0\right)$ and $\max \left(\gamma_{T}-\right.$ $\left.\delta_{T}, \gamma_{H}-\delta_{H}, 0\right)$, respectively. Since all other terms in $\Delta F_{T}$ and $\Delta F_{H}$ are rank one matrices, the rank of $\triangle F$ is bounded above by

$$
\beta_{T}+\beta_{H}+\delta_{T}+\delta_{H}+\max \left(\alpha_{T}-\beta_{T}, \alpha_{H}-\beta_{H}, 0\right)+\max \left(\gamma_{T}-\delta_{T}, \gamma_{H}-\delta_{H}, 0\right)
$$

which is the same as $\eta$ given in (3.11) with $\eta_{c}=0$.

Now, suppose that $B_{T}(z) D_{T}(z)$ and $B_{H}(z) D_{H}(z)$ have $\eta_{c}$ common roots. This implies four combinations. That is, $B_{T}(z)$ and $B_{H}(z), B_{T}(z)$ and $D_{H}(z), D_{T}(z)$ and $B_{H}(z)$, or $D_{T}(z)$ and $D_{H}(z)$ have common roots. Without loss of generality, we assume that $B_{T}(z)$ and $B_{H}(z)$ have a common root $\mu$ and that Toeplitz matrices $T_{1, k}$ 
and $J H_{1, k}$ are generated by this root. By postmultiplying $F_{T, 1, k}, F_{H, 1, k}, F_{T, 1, k}+$ $J F_{H, 1, k}$ with the lower triangular Toeplitz matrix $L_{\mu}$ which has

$$
[1,-\mu, 0, \ldots, 0]^{T}
$$

as the first column, the resulting matrices have only one nonzero column. Thus, the rank of $\triangle F=\triangle F_{T}+J \triangle F_{H}$ is lower than (3.12) by one due to the root $\mu$ shared by $B_{T}(z)$ and $B_{H}(z)$.

As to the other three combinations, similar arguments hold with the following modifications. When $D_{T}(z)$ and $D_{H}(z)$ have common roots, we postmultiply $F_{T, 2, k}+$ $J F_{H, 2, k}$ by the corresponding upper triangular banded Toeplitz matrix constructed with respect to the common root. When $B_{T}(z)$ and $D_{H}(z)$ (or $B_{H}(z)$ and $D_{T}(z)$ ) have common roots, we examine the rank of

$$
\triangle F_{T, 1, k}+J \triangle F_{H, 2, k} J J=\triangle F_{T, 1, k}+\Delta F_{H, 2, k}^{T} J
$$

by premultiplying an appropriate lower (or upper) triangular Toeplitz banded matrix.

Let us now focus on the case that $B_{T}(z), D_{T}(z), B_{H}(z)$, or $D_{H}(z)$ has repeated roots which are common for both $B_{T}(z) D_{T}(z)$ and $B_{H}(z) D_{H}(z)$. Without loss of generality, we assume that $B_{T}(z)$ has $k_{T}$ roots at $\mu$ and that $B_{H}(z)$ has $k_{H}$ roots at $\mu$. Let $F_{T, 1, i}, 1 \leq i \leq k_{T}$, and $F_{H, 1, j}, 1 \leq j \leq k_{H}$, be constructed with respect to the repeated roots $\mu$. It can be shown that

$$
\left(\sum_{i=1}^{k_{T}} F_{T, 1, i}+\sum_{j=1}^{k_{H}} F_{H, 1, i}\right) L_{\mu}^{k}, \quad k=\max \left(k_{T}, k_{H}\right),
$$

has at most $k$ nonzero columns. Therefore, the rank of $\Delta F=\Delta F_{T}+J \triangle F_{H}$ is lower than (3.12) by $\min \left(k_{T}, k_{H}\right)$ due to the repeated roots shared by $B_{T}(z)$ and $B_{H}(z)$.

3.3. The clustering radius of $P^{-1} A$. It is clear from the analysis in $\S 3.2$ that the number of outliers of $P^{-1} A$ does not depend on the boundedness of $\left\|A^{-1}\right\|_{2}$. To examine the spectral clustering property of $P^{-1} A$, we restrict our discussion in this subsection to the case that $\left\|A^{-1}\right\|_{2}$ exists and is bounded by a constant independent of the size $N$ of $A$. Besides, it is also assumed that $B_{T}(z) D_{T}(z)$ and $B_{H}(z) D_{H}(z)$ have no common roots $\left(\eta_{c}=0\right)$.

Since nonzero eigenvalues of $P^{-1} A$ and $A^{-1} \triangle A$ are related via

$$
\left[\lambda\left(P^{-1} A\right)\right]^{-1}=\lambda\left(A^{-1}(A+\triangle A)\right)=1+\lambda\left(A^{-1} \triangle A\right),
$$

the eigenvalues of $P^{-1} A$ clustered around unity are equivalent to the eigenvalues of $A^{-1} \triangle A$ clustered around zero. Besides, it is known from the proof of Theorem 2 that $\triangle A$ is asymptotically equivalent to

$$
\triangle F=\triangle F_{T}+J \Delta F_{H}=\left(F_{T, 1}+F_{T, 2}\right)+J\left(F_{H, 1}+F_{H, 2}\right),
$$

at a rate of $O\left(\left|t_{N}\right|+\left|t_{-N}\right|+\left|h_{N}\right|+\left|h_{-N}\right|\right)$. Thus, we divide the discussion on the spectral clustering radius of $A^{-1} \triangle A$ into two steps.

Step 1. Transform $A$ and $\triangle F$ into $Q_{A}$ and $\triangle Q_{F}$ whose eigenstructures are easier to analyze (Lemmas 3-5).

Step 2. Use the perturbation theory to determine the spectral clustering radius of $A^{-1} \triangle A$ or, equivalently, $P^{-1} A$ (Lemmas 6 and 7 , and Theorem 3 ). 
We first transform the generalized eigenvalue problem

$$
\triangle F \mathbf{x}=\lambda A \mathbf{x}, \quad \Delta F=\triangle F_{T}+J \Delta F_{H}, \quad A=T+J T_{H},
$$

to another generalized eigenvalue problem

$$
\triangle Q_{F} \tilde{\mathbf{x}}=\lambda Q_{A} \tilde{\mathbf{x}}, \quad \mathbf{x}=U_{D, T} U_{D, H} L_{B, T} L_{B, H} \tilde{\mathbf{x}}
$$

where

$$
\begin{aligned}
\triangle Q_{F} & =\left(L_{B, T} \mathcal{U}_{B, H}^{T}\right) \Delta F\left(U_{D, T} U_{D, H} L_{B, T} L_{B, H}\right), \\
Q_{A} & =\left(L_{B, T} \mathcal{U}_{B, H}^{T}\right) A\left(U_{D, T} U_{D, H} L_{B, T} L_{B, H}\right) .
\end{aligned}
$$

In (3.15a) and (3.15b), $\mathcal{U}_{B, H}$ is an $N \times N$ upper triangular Toeplitz matrices generated by $z^{\beta_{H}} B_{H}\left(z^{-1}\right)$, whereas all other matrices are defined in (3.5). The postmultiplication with $U_{D, T} U_{D, H} L_{B, T} L_{B, H}$ in (3.15a) is to make the elements of $\triangle Q_{F}$ zero except the first and last several columns as described in Lemma 3. The premultiplication with $L_{B, T} \mathcal{U}_{B, H}^{T}$ and the postmultiplication with $U_{D, T} U_{D, H}$ in $(3.15 \mathrm{~b})$ is used to simplify the structure of $Q_{A}$ so that the elements in the four corner blocks of $Q_{A}$ do not change with $N$, as shown in Lemma 4. An explanation for introducing the matrix $\mathcal{U}_{B, H}$ will be given after the proof of Lemma 4 .

Lemma 3. Let $T$ and $H$ be $N \times N$ Toeplitz and Hankel matrices generated by $T(z)$ and $H(z)$ in (3.3) with the corresponding generating sequences satisfying (3.1) and (3.2). The elements of $\triangle Q_{F}$ are zeros except the first $s_{0}$ and last $r_{0}$ columns, where

$$
s_{0}=\max \left(s_{T}+\delta_{H}, s_{H}+\delta_{T}\right), \quad r_{0}=\max \left(r_{T}+\beta_{H}, r_{H}+\beta_{T}\right)
$$

Proof. Recall from (3.9) that $\Delta F_{T}=F_{T, 1}+F_{T, 2}$ and

$$
\left(F_{T, 1}\right)_{i, j}=t_{N+i-j}, \quad\left(F_{T, 2}\right)_{i, j}=t_{-N+i-j} .
$$

The $(i, j)$ element of $F_{T, 1} U_{D, T} U_{D, H} L_{B, T} L_{B, H}$ is

$$
\sum_{k=1}^{N} \sum_{l=1}^{N} \sum_{m=1}^{N} \sum_{n=1}^{N} t_{N+i-k} d_{T, l-k} d_{H, m-l} b_{T, m-n} b_{H, n-j} .
$$

If $j \leq N-\left(r_{T}+\beta_{H}\right)$, the above summation can be simplified as

$$
\sum_{k^{\prime}=0}^{\delta_{T}} \sum_{l^{\prime}=0}^{\delta_{H}} \sum_{n^{\prime}=0}^{\beta_{H}}\left(\sum_{m^{\prime}=0}^{\beta_{T}} t_{N+i+k^{\prime}+l^{\prime}-m^{\prime}-n^{\prime}-j} b_{T, m^{\prime}}\right) d_{T, k^{\prime}} d_{H, l^{\prime}} b_{H, n^{\prime}}=0
$$

where $k^{\prime}=l-k, l^{\prime}=m-l, m^{\prime}=m-n, n^{\prime}=n-j$, and the equality is due to (3.8). Similarly, the $(i, j)$ element of $F_{T, 2} U_{D, T} U_{D, H} L_{B, T} L_{B, H}$ can be simplified as

$$
\sum_{l^{\prime}=0}^{\delta_{H}} \sum_{m^{\prime}=0}^{\delta_{T}} \sum_{n^{\prime}=0}^{\beta_{H}}\left(\sum_{k^{\prime}=0}^{\delta_{T}} t_{-N+i+k^{\prime}+l^{\prime}-m^{\prime}-n^{\prime}-j} d_{T, k^{\prime}}\right) d_{H, l^{\prime}} b_{T, m^{\prime}} b_{H, n^{\prime}}=0,
$$

for $j>s_{T}+\delta_{H}$. Thus, the elements of $\triangle F_{T} U_{D, T} U_{D, H} L_{B, T} L_{B, H}$ are zeros except the first $s_{T}+\delta_{H}$ and last $r_{T}+\beta_{H}$ columns. We can argue in a similar fashion that 
the elements of $\triangle F_{H} U_{D, T} U_{D, H} L_{B, T} L_{B, H}$ are zeros except the first $s_{H}+\delta_{T}$ and last $r_{H}+\beta_{T}$ columns. Then, it is clear that the elements of $\triangle Q_{F}$ are zeros except the first $s_{0}$ and last $r_{0}$ columns, and the proof is completed.

According to Lemma 3, the rank space of $Q_{A}^{-1} \triangle Q_{F}$ is contained in

$$
\mathcal{R}=\left\{\mathbf{v} \in R^{N} \mid v_{n}=0, s_{0}<n \leq N-r_{0}\right\}
$$

Therefore, the nonzero eigenvalues of $Q_{A}^{-1} \triangle Q_{F}$ only depend on the northwest $s_{0} \times s_{0}$, the northeast $s_{0} \times r_{0}$, the southwest $r_{0} \times s_{0}$, and the southeast $r_{0} \times r_{0}$ blocks of $Q_{A}$ and $\triangle Q_{F}$. The structures of the four corner blocks of $Q_{A}$ and $\triangle Q_{F}$ are stated in Lemmas 4 and 5 , respectively.

LeMmA 4. Let $T$ and $H$ be $N \times N$ Toeplitz and Hankel matrices generated by $T(z)$ and $H(z)$ in (3.3) with the corresponding generating sequences satisfying (3.1) and (3.2). The elements in the northwest $s_{0} \times s_{0}$, northeast $s_{0} \times r_{0}$, southwest $r_{0} \times s_{0}$, and southeast $r_{0} \times r_{0}$ blocks of $Q_{A}$ do not change for sufficiently large $N$.

Proof. With the relation $A=T+J T_{H}$, the $Q_{A}$ can be written as

$$
Q_{A}=Q_{T}+Q_{H}
$$

where

$$
\begin{aligned}
Q_{T} & =\left(L_{B, T} \mathcal{U}_{B, H}^{T}\right) T\left(U_{D, T} U_{D, H} L_{B, T} L_{B, H}\right), \\
Q_{H} & =\left(L_{B, T} \mathcal{U}_{B, H}^{T}\right) J T_{H}\left(U_{D, T} U_{D, H} L_{B, T} L_{B, H}\right) .
\end{aligned}
$$

By using (3.5), the fact that $\mathcal{U}_{B, H}^{T}$ is a lower triangular Toeplitz matrix, and the commutative property of the matrix product among lower (or upper) triangular Toeplitz matrices, we obtain

$$
Q_{T}=\mathcal{U}_{B, H}^{T}\left(L_{A, T} U_{D, T}+L_{B, T} U_{C, T}\right) U_{D, H} L_{B, T} L_{B, H},
$$

which is a product of lower and upper triangular banded Toeplitz matrices. Thus, the elements in the corresponding four corner blocks of $Q_{T}$ do not change for sufficiently large $N$.

To examine elements in the four corner blocks of $Q_{H}$, we use the relation

$$
L_{B, T} \mathcal{U}_{B, H}^{T} J=J J L_{B, T} J J \mathcal{U}_{B, H}^{T} J=J L_{B, T}^{T} \mathcal{U}_{B, H},
$$

and rewrite $Q_{H}$ as

$$
Q_{H}=J L_{B, T}^{T}\left(\mathcal{U}_{B, H} T_{H} U_{D, H}\right) U_{D, T} L_{B, T} L_{B, H}
$$

Let us decompose $\mathcal{U}_{B, H}$ into matrices consisting of its north and south blocks, respectively, i.e.,

$$
\mathcal{U}_{B, H}=W_{N}+W_{S},
$$

where $W_{N}$ (or $W_{S}$ ) consists of the first $N-\beta_{H}$ (or last $\beta_{H}$ ) rows of $\mathcal{U}_{B, H}$ and zeros elsewhere. Since the first $N-\beta_{H}$ rows of $\mathcal{U}_{B, H}$ are the same as the last $N-\beta_{H}$ rows of $L_{B, H}$, we know that

$$
\tilde{W}_{N}=\left(W_{N} T_{H} U_{D, H}\right) U_{D, T} L_{B, T} L_{B, H}
$$


has its last $\beta_{H}$ rows equal to zero and first $N-\beta_{H}$ rows equal to the last $N-\beta_{H}$ rows of

$$
\begin{aligned}
G & =\left(L_{B, H} T_{H} U_{D, H}\right) U_{D, T} L_{B, T} L_{B, H} \\
& =\left(L_{A, H} U_{D, H}+L_{B, H} U_{C, H}\right) U_{D, T} L_{B, T} L_{B, H} .
\end{aligned}
$$

The elements in the four corner blocks of $J L_{B, T}^{T} G$ do not change for sufficiently large $N$ since it is a product of lower and upper banded triangular Toeplitz matrices. Consequently, the elements in the corresponding four corner blocks of

$$
J L_{B, T}^{T} \tilde{W}_{N}=J L_{B, T}^{T}\left(W_{N} T_{H} U_{D, H}\right) U_{D, T} L_{B, T} L_{B, H}
$$

do not change with $N$. Besides, since $\mathcal{U}_{B, H}$ is upper-triangular, the elements of $W_{S}=\mathcal{U}_{B, H}-W_{N}$ are zeros except the southeast $\beta_{H} \times \beta_{H}$ block. It can be shown directly by matrix multiplication that the elements in the four corner blocks of

$$
J L_{B, T}^{T}\left(W_{S} T_{H} U_{D, H}\right) U_{D, T} L_{B, T} L_{B, H}
$$

do not change with $N$, and the proof is completed.

With the above proof, it is easier to explain the need for introducing the premultiplying matrix $\mathcal{U}_{B, H}^{T}$ in transformation (3.15). First, it has to be a lower triangular Toeplitz so that it commutes with $L_{B, T}$. Second, in order to move the time-reversal operator $J$ in $Q_{H}$ to the front as in (3.18), its transpose has to behave similarly as $L_{B, H}$ so that its multiplication with $J L_{B, H}^{-1}$ relates to a banded matrix. The $\mathcal{U}_{B, H}^{T}$ satisfies both conditions, and is then used in transformation (3.15).

Lemma 5. Let $T$ and $H$ be $N \times N$ Toeplitz and Hankel matrices generated by $T(z)$ and $H(z)$ in (3.3) with the corresponding generating sequences satisfying (3.1) and (3.2). The corresponding four corner blocks of $\triangle Q_{F}$ converge to $\bar{Q}_{N W}, \bar{Q}_{N E}$, $\bar{Q}_{S W}$, and $\bar{Q}_{S E}$, respectively, where $\bar{Q}_{N E}$ is the the northeast $s_{0} \times r_{0}$ block of

$$
\left(L_{B, T} \mathcal{U}_{B, H}^{T}\right) F_{T, 1}\left(U_{D, T} U_{D, H} L_{B, T} L_{B, H}\right),
$$

$\bar{Q}_{S W}$ is the the southwest $r_{0} \times s_{0}$ block of

$$
\left(L_{B, T} \mathcal{U}_{B, H}^{T}\right) F_{T, 2}\left(U_{D, T} U_{D, H} L_{B, T} L_{B, H}\right),
$$

$\bar{Q}_{N W}$ is the the northwest $s_{0} \times s_{0}$ block of

$$
\left(L_{B, T} \mathcal{U}_{B, H}^{T}\right) J F_{H, 2}\left(U_{D, T} U_{D, H} L_{B, T} L_{B, H}\right),
$$

and $\bar{Q}_{S E}$ is the the southeast $r_{0} \times r_{0}$ block of

$$
\left(L_{B, T} \mathcal{U}_{B, H}^{T}\right) J F_{H, 1}\left(U_{D, T} U_{D, H} L_{B, T} L_{B, H}\right) .
$$

The elements of $\bar{Q}_{N W}, \bar{Q}_{N E}, \bar{Q}_{S W}$, and $\bar{Q}_{S E}$ do not change for sufficiently large $N$.

Proof. Recall that

$$
\triangle F=\triangle F_{T}+J \triangle F_{H}=\left(F_{T, 1}+F_{T, 2}\right)+J\left(F_{H, 1}+F_{H, 2}\right) .
$$

Let us define

$$
\tilde{T}_{1}=F_{T, 1} U_{D, T} U_{D, H} L_{B, T} L_{B, H} \quad \text { and } \quad \tilde{T}_{2}=F_{T, 2} U_{D, T} U_{D, H} L_{B, T} L_{B, H} .
$$


With (3.16), the $(i, j)$ element of $\tilde{T}_{1}$ is bounded by

$$
\begin{aligned}
& \sum_{k^{\prime}=0}^{\delta_{T}} \sum_{l^{\prime}=0}^{\delta_{H}} \sum_{m^{\prime}=0}^{\beta_{T}} \sum_{n^{\prime}=0}^{\beta_{H}}\left|t_{N+i+k^{\prime}+l^{\prime}-m^{\prime}-n^{\prime}-j}\right|\left|d_{T, k^{\prime}}\right|\left|d_{H, l^{\prime}}\right|\left|b_{T, m^{\prime}}\right|\left|b_{H, n^{\prime}}\right| \\
& \quad \leq \sum_{m^{\prime}=0}^{\beta_{T}}\left|b_{T, m^{\prime}}\right| \sum_{n^{\prime}=0}^{\beta_{H}}\left|b_{H, n^{\prime}}\right| \sum_{k^{\prime}=0}^{\delta_{T}}\left|d_{T, k^{\prime}}\right| \sum_{l^{\prime}=0}^{\delta_{H}}\left|d_{H, l^{\prime}}\right| \\
& \max _{-\left(\beta_{T}+\beta_{H}\right) \leq n \leq\left(\delta_{T}+\delta_{H}\right)}\left|t_{N+i-j+n}\right| .
\end{aligned}
$$

To determine an upper bound for $\sum_{m=0}^{\beta_{T}}\left|b_{T, m}\right|$, we factorize $B_{T}\left(z^{-1}\right)$ as

$$
B_{T}\left(z^{-1}\right)=\left(1-r_{1} z^{-1}\right)\left(1-r_{2} z^{-1}\right) \cdots\left(1-r_{\beta_{T}} z^{-1}\right) \text {. }
$$

A direct consequence of (3.1) is that $\left|r_{i}\right|<1,1 \leq i \leq \beta_{T}$, so that

$$
\left|b_{T, m}\right| \leq\left(\begin{array}{c}
\beta_{T} \\
m
\end{array}\right)\left(\max \left|r_{i}\right|\right)^{m} \leq\left(\begin{array}{c}
\beta_{T} \\
m
\end{array}\right), \quad \text { where } \quad\left(\begin{array}{c}
\beta_{T} \\
m
\end{array}\right) \equiv \frac{\beta_{T} !}{\left(\beta_{T}-m\right) ! m !}
$$

Therefore, we obtain

$$
\sum_{m=0}^{\beta_{T}}\left|b_{T, m}\right| \leq \sum_{m=0}^{\beta_{T}}\left(\begin{array}{c}
\beta_{T} \\
m
\end{array}\right)=2^{\beta_{T}} .
$$

Similarly, we can derive that

$$
\sum_{l=0}^{\beta_{H}}\left|b_{H, l}\right| \leq 2^{\beta_{H}}, \quad \sum_{k=0}^{\delta_{T}}\left|d_{T, k}\right| \leq 2^{\delta_{T}}, \quad \sum_{l=0}^{\delta_{H}}\left|d_{H, l}\right| \leq 2^{\delta_{H}} .
$$

Thus, the $(i, j)$ element of $\tilde{T}_{1}$ is bounded by

$$
2^{\beta_{T}+\beta_{H}+\delta_{T}+\delta_{H}} \max _{-\left(\beta_{T}+\beta_{H}\right) \leq n \leq\left(\delta_{T}+\delta_{H}\right)}\left|t_{N+i-j+n}\right| .
$$

We can see from (3.22) that the elements in the corresponding northeast, northwest, southeast, and southwest blocks of $\tilde{T}_{1}$ are bounded by $O\left(\left|t_{0}\right|\right), O\left(\left|t_{N}\right|\right), O\left(\left|t_{N}\right|\right)$, and $O\left(\left|t_{2 N}\right|\right)$, respectively. The same result holds for $L_{B, T} \mathcal{U}_{B, H}^{T} \tilde{T}_{1}$, since $L_{B, T} \mathcal{U}_{B, H}^{T}$ is a lower triangular banded Toeplitz matrix with 2-norm of $O(1)$. In addition, we can verify that the elements in the northeast block of $L_{B, T} \mathcal{U}_{B, H}^{T} \tilde{T}_{1}$ (i.e., $\bar{Q}_{N E}$ ) are fixed for sufficiently large $N$, which is due to the banded structures of $\mathcal{U}_{B, H}^{T}, U_{D, T}, U_{D, H}$, $L_{B, T}$, and $L_{B, H}$. Similarly, we can argue that the elements in the corresponding northeast, northwest, southeast, and southwest blocks of $L_{B, T} \mathcal{U}_{B, H}^{T} \tilde{T}_{2}$ are bounded by $O\left(\left|t_{-2 N}\right|\right), O\left(\left|t_{-N}\right|\right), O\left(\left|t_{-N}\right|\right)$, and $O\left(\left|t_{0}\right|\right)$, respectively, and the elements in the southwest corner block (i.e., $\bar{Q}_{S W}$ ) do not change for large $N$. Since $T$ and $T_{H}$ are both rational Toeplitz, the same results also hold for

$\left(L_{B, T} \mathcal{U}_{B, H}^{T}\right)\left(J F_{H, 1} U_{D, T} U_{D, H} L_{B, T} L_{B, H}\right), \quad\left(L_{B, T} \mathcal{U}_{B, H}^{T}\right)\left(J F_{H, 2} U_{D, T} U_{D, H} L_{B, T} L_{B, H}\right)$, and the proof is completed.

With the above lemma, we change the corresponding four corner blocks of $\triangle Q_{F}$ to their asymptotic values, and denote the resulting matrix by $\triangle \bar{Q}_{F}$, i.e.,

$$
\triangle \bar{Q}_{F}=\left[\begin{array}{ccc}
\bar{Q}_{N W} & 0 & \bar{Q}_{N E} \\
Q_{W} & 0 & Q_{E} \\
\bar{Q}_{S W} & 0 & \bar{Q}_{S E}
\end{array}\right]
$$


where $Q_{W}$ (or $\left.Q_{E}\right)$ are the middle $N-\left(r_{0}+s_{0}\right)$ rows in the first $s_{0}$ (or the last $r_{0}$ ) columns of $\triangle Q_{F}$. Thus, the matrix $\triangle Q_{F}-\Delta \bar{Q}_{F}$ converges to the zero matrix at a rate bounded by

$$
\left\|\triangle Q_{F}-\triangle \bar{Q}_{F}\right\|_{2} \leq O\left(\left|t_{N}\right|+\left|t_{-N}\right|+\left|h_{N}\right|+\left|h_{-N}\right|\right) .
$$

Since the nonzero eigenvalues of $Q_{A}^{-1} \triangle \bar{Q}_{F}$ only depend on elements in the corresponding four corner blocks of $Q_{A}$ and $\triangle \bar{Q}_{F}$, which do not change with $N$, the framework of the perturbation analysis for eigenvalues can be conveniently applied as described in the following.

Due to transformation (3.15), the eigenvalues of $A^{-1} \triangle A$ are those of $Q_{A}^{-1} \triangle Q_{A}$ where

$$
\triangle Q_{A}=\left(L_{B, T} \mathcal{U}_{B, H}^{T}\right) \triangle A\left(U_{D, T} U_{D, H} L_{B, T} L_{B, H}\right) .
$$

We can view $\triangle Q_{A}$ as the sum of $\triangle \bar{Q}_{F}$ and a perturbation matrix

$$
\triangle Q_{E} \equiv \Delta Q_{A}-\Delta \bar{Q}_{F}=\left(\Delta Q_{A}-\Delta Q_{F}\right)+\left(\Delta Q_{F}-\Delta \bar{Q}_{F}\right) .
$$

To determine a bound on $\left\|\triangle Q_{E}\right\|_{2}$, we have

$$
\left\|\triangle Q_{E}\right\|_{2} \leq\left\|\triangle Q_{A}-\triangle Q_{F}\right\|_{2}+\left\|\triangle Q_{F}-\triangle \bar{Q}_{F}\right\|_{2} .
$$

By using Lemma 2 and the boundedness of

$$
\left\|U_{D, T}\right\|_{2},\left\|U_{D, H}\right\|_{2},\left\|L_{B, T}\right\|_{2},\left\|L_{B, H}\right\|_{2}, \quad \text { and }\left\|\mathcal{U}_{B, H}^{T}\right\|_{2}
$$

which is implied from (3.20) and (3.21), it is clear that

$$
|| \triangle Q_{A}-\triangle Q_{F} \|_{2} \leq O\left(\left|t_{N}\right|+\left|t_{-N}\right|+\left|h_{N}\right|+\left|h_{-N}\right|\right) .
$$

The above equation, (3.24), and (3.25), give a bound on $\left\|\triangle Q_{E}\right\|_{2}$, i.e.,

$$
\left\|\triangle Q_{E}\right\|_{2} \leq O\left(\left|t_{N}\right|+\left|t_{-N}\right|+\left|h_{N}\right|+\left|h_{-N}\right|\right) .
$$

In addition, we know from (3.15) that

$$
\left\|Q_{A}^{-1}\right\|_{2} \leq\left\|L_{B, H}^{-1}\right\|_{2}\left\|L_{B, T}^{-1}\right\|_{2}\left\|U_{D, H}^{-1}\right\|_{2}\left\|U_{D, T}^{-1}\right\|_{2}\left\|A^{-1}\right\|_{2}\left\|\mathcal{U}_{B, H}^{-T}\right\|_{2}\left\|L_{B, T}^{-1}\right\|_{2} .
$$

Since all roots of the generating function for lower (or upper) triangular banded matrices in the above inequality are inside (or outside) the unit circle due to (3.1), all matrix norms in the right-hand side of (3.26) are bounded by a constant independent of $N$ [23], [25]. Therefore, $\left\|Q_{A}^{-1}\right\|_{2}=O(1)$ and we obtain the following lemma.

Lemma 6. Let $T$ and $H$ be $N \times N$ Toeplitz and Hankel matrices generated by $T(z)$ and $H(z)$ in (3.3) with the corresponding generating sequences satisfying (3.1) and (3.2). Then

$$
\left\|Q_{A}^{-1} \triangle Q_{E}\right\|_{2} \leq \varepsilon=O\left(\left|t_{N}\right|+\left|t_{-N}\right|+\left|h_{N}\right|+\left|h_{-N}\right|\right)
$$

for sufficiently large $N$.

Note that $\triangle \bar{Q}_{F}$ has at most $r_{0}+s_{0}$ nonzero columns, which is equal to $\eta$ in (3.10) with $\eta_{c}=0$. The rank $\eta_{r}$ of $\Delta \bar{Q}_{F}$ is therefore bounded by

$$
\eta_{r} \leq \eta \leq \tilde{\eta}
$$


where $\eta$ and $\tilde{\eta}$ are defined in (3.10) and (3.11). We arrange the eigenvalues of $Q_{A}^{-1} \triangle \bar{Q}_{F}$ in descending order, i.e., $\left|\lambda_{n}\right| \geq\left|\lambda_{n+1}\right|$, and denote the corresponding normalized right-hand and left-hand eigenvectors by $\mathbf{x}_{1}, \mathbf{x}_{2}, \ldots, \mathbf{x}_{N}$ and $\mathbf{y}_{1}, \mathbf{y}_{2}, \ldots, \mathbf{y}_{N}$, respectively. Since the rank of $\Delta \bar{Q}_{F}$ is $\eta_{r}, \lambda_{n}=0$ for $\eta_{r}<n \leq N$. We choose vectors $\mathbf{x}_{n}$ with $\eta_{r}<n \leq N$ to be orthogonal for different values of $n$. We also define

$$
s_{n}=\mathbf{y}_{n}^{H} \mathbf{x}_{n}, \quad 1 \leq n \leq N .
$$

The reciprocal of $s_{n}$ is usually known as the condition of the eigenvalue $\lambda_{n}$ [15], which is bounded uniformly by a constant independent of $N$ as stated in Lemma 7 .

Lemma 7. Let $T$ and $H$ be $N \times N$ Toeplitz and Hankel matrices generated by $T(z)$ and $H(z)$ in (3.3) with the corresponding generating sequences satisfying (3.1) and (3.2). Then, the $\left|s_{m}^{-1}\right|, 1 \leq m \leq \eta_{r}$, of $Q_{A}^{-1} \triangle \bar{Q}_{F}$ is bounded by a constant independent of $N$.

Proof. According to (3.23), it is clear that the rank space of $Q_{A}^{-1} \triangle \bar{Q}_{F}$ is contained in

$$
\mathcal{R}=\left\{\mathbf{v} \in R^{N} \mid v_{n}=0, s_{0}<n \leq N-r_{0}\right\}
$$

where

$$
s_{0}=\max \left(s_{T}+\delta_{H}, s_{H}+\delta_{T}\right), \quad r_{0}=\max \left(r_{T}+\beta_{H}, r_{H}+\beta_{T}\right) .
$$

All nonzero eigenvalues of $Q_{A}^{-1} \triangle \bar{Q}_{F}$ can be determined by considering the generalized eigenvalue problem in the subspace $\mathcal{R}$. Since all elements in the corresponding four corner blocks of $Q_{A}$ and $\triangle \bar{Q}_{F}$ do not change with $N$, the boundedness of $\left|s_{m}^{-1}\right|$, $1 \leq m \leq \eta_{r}$, is implied, and the proof is completed.

The eigenvalues of

$$
Q_{A}^{-1} \triangle Q_{A}=Q_{A}^{-1} \triangle \bar{Q}_{F}+Q_{A}^{-1} \triangle Q_{E}
$$

can be estimated from those of $Q_{A}^{-1} \triangle \bar{Q}_{F}$ through perturbation theory. Since the norm of the perturbation matrix is equal to $\varepsilon$ as given in Lemma 6, we denote the eigenvalues and the right-hand eigenvectors of the perturbed matrix $Q_{A}^{-1} \triangle Q_{A}$ by $\lambda_{n}(\varepsilon)$ and $\mathbf{x}_{n}(\varepsilon)$, respectively. According to perturbation theory for repeated eigenvalues [33], the eigenvectors $\mathbf{x}_{n}(\varepsilon)$ with $\eta_{r}<n \leq N$ must take the form

$$
\mathbf{x}_{n}(\varepsilon)=\sum_{m=1}^{\eta_{r}} \frac{\xi_{m n}}{\left(\lambda_{n}-\lambda_{m}\right) s_{m}} \mathbf{x}_{m}+\sum_{m=\eta_{r}+1}^{N} g_{m n} \mathbf{x}_{m}+O\left(\varepsilon^{2}\right)
$$

where $\xi_{m n}=\mathbf{y}_{m}^{H} Q_{A}^{-1} \triangle Q_{E} \mathbf{x}_{n}, \lambda_{n}=0, g_{n n}=1$, and $s_{m}$ defined in (3.28). Due to the construction, we know that

$$
\left\|\mathbf{x}_{n}(\varepsilon)\right\|_{2} \geq\left\|\mathbf{x}_{n}\right\|_{2}=1
$$

The factor $\left|\xi_{m n}\right|$ is bounded by

$$
\left|\xi_{m n}\right|=\left|\mathbf{y}_{m}^{H} Q_{A}^{-1} \triangle Q_{E} \mathbf{x}_{n}\right| \leq\left\|\mathbf{y}_{m}\right\|_{2}\left\|Q_{A}^{-1} \triangle Q_{E}\right\|_{2}|| \mathbf{x}_{n} \|_{2} \leq \varepsilon .
$$

The $\left|s_{m}^{-1}\right|, 1 \leq m \leq \eta_{r}$, is also bounded due to Lemma 7. The magnitude of $\lambda_{n}(\varepsilon)$, $\eta_{r}<n \leq N$, of $Q_{A}^{-1} \triangle Q_{A}$ is approximated by

$$
\left|\lambda_{n}(\varepsilon)\right|=\frac{\left\|Q_{A}^{-1}\left(\triangle \bar{Q}_{F}+\triangle Q_{E}\right) \mathbf{x}_{n}(\varepsilon)\right\|_{2}}{\left\|\mathbf{x}_{n}(\varepsilon)\right\|_{2}}
$$




$$
\begin{aligned}
& \leq \sum_{m=1}^{\eta_{r}} \frac{\left\|\xi_{m n} Q_{A}^{-1} \triangle \bar{Q}_{F} \mathbf{x}_{m}\right\|_{2}}{\left|\lambda_{m} s_{m}\right| \mid\left\|\mathbf{x}_{n}(\varepsilon)\right\|_{2}}+\left\|Q_{A}^{-1} \triangle Q_{E}\right\|_{2} \\
& \leq \sum_{m=1}^{\eta_{r}} \frac{\varepsilon}{\left|s_{m}\right|}+\varepsilon .
\end{aligned}
$$

Since $\eta_{r} \leq \eta$ as given by (3.27), we conclude that $Q_{A}^{-1} \triangle Q_{A}$ (or $A^{-1} \triangle A$ ) has at least $N-\eta$ eigenvalues bounded by

$$
\epsilon_{Q}=O\left(\left|t_{N}\right|+\left|t_{-N}\right|+\left|h_{N}\right|+\left|h_{-N}\right|\right)
$$

for sufficiently large $N$. With (3.13), the above results are summarized in the following theorem.

Theorem 3. Let $T$ and $H$ be $N \times N$ Toeplitz and Hankel matrices generated by $T(z)$ and $H(z)$ in (3.3) with the corresponding generating sequences satisfying (3.1) and (3.2). Besides, $A=T+H$ has a bounded inverse, and $B_{T}(z) D_{T}(z)$ and $B_{H}(z) D_{H}(z)$ have no common roots. For sufficiently large $N$, there are at least $N-\eta$ eigenvalues of $P^{-1} A$, where $\eta$ is given in (3.11) with $\eta_{c}=0$, confined in the disk centered at unity with radius $\epsilon$ proportional to

$$
\epsilon=O\left(\left|t_{N}\right|+\left|t_{-N}\right|+\left|h_{N}\right|+\left|h_{-N}\right|\right) .
$$

4. Numerical experiments. We use four test problems, including symmetric and nonsymmetric $T$ and $T_{H}$, to illustrate the analysis. For all Toeplitz-plus-Hankel systems $A \mathbf{x}=\mathbf{b}$ to be solved in the experiments, we choose $\mathbf{b}=(1, \ldots, 1)^{T}$ and the zero initial guess.

Test Problem 1. Symmetric $T$ and $T_{H}$ with $\left(\alpha_{T}, \beta_{T}, \gamma_{T}, \delta_{T}\right)=(1,1,1,1)$ and $\left(\alpha_{H}, \beta_{H}, \gamma_{H}, \delta_{H}\right)=(1,2,1,2)$.

The generating functions of $T$ and $T_{H}$ are chosen to be

$$
\begin{aligned}
& T(z)=\frac{0.5+0.7 z^{-1}}{1+0.7 z^{-1}}+\frac{0.5+0.7 z}{1+0.7 z} \\
& H(z)=\frac{0.5-0.4 z^{-1}}{\left(1-0.7 z^{-1}\right)\left(1-0.9 z^{-1}\right)}+\frac{0.5-0.4 z}{(1-0.7 z)(1-0.9 z)}
\end{aligned}
$$

The eigenvalues of $A^{-1} \triangle A$, except those with magnitude less than $10^{-6}$, are plotted in Fig. 1. Although it is difficult to distinguish the outliers from the clustered eigenvalues for $N \leq 64$, we can see six outliers more easily for the case $N=128$. The number of outliers is consistent with (3.11), where the last three terms are all equal to zero. The clustering radii $\epsilon,\left|t_{N}\right|$, and $\left|h_{N}\right|$ for different $N$ are listed in Table 1 . The values of $\epsilon$ decrease at a rate of $O\left(\left|t_{N}\right|+\left|h_{N}\right|\right)$. The convergence history of the CG and PCG methods are plotted in Fig. 2. The upper four curves are those of the CG method whereas the lower four curves correspond to those of the PCG method. The preconditioning does accelerate the convergence rate of the CG method significantly. The convergence rate of the CG method becomes slower for larger $N$. In contrast, the PCG method converges faster as $N$ becomes larger. It in fact converges in four $(=\eta / 2+1)$ iterations asymptotically. The reason that it takes only $\eta / 2+1$ iterations for the PCG method to converge can be explained by the fact that that the outliers are related in pairs such that only $\eta / 2$ iterations are needed to eliminate the effects 
TABLE 1

The clustering radius $\epsilon$ of $P^{-1} A$ for Test Problem 1.

\begin{tabular}{|c||c|c|c|}
\hline$N$ & $\left|t_{N}\right|=\left|t_{-N}\right|$ & $\left|h_{N}\right|=\left|h_{-N}\right|$ & $\epsilon$ \\
\hline \hline 16 & $1.6 \times 10^{-3}$ & $4.7 \times 10^{-2}$ & $1.5 \times 10^{-1}$ \\
\hline 32 & $5.5 \times 10^{-6}$ & $8.6 \times 10^{-3}$ & $8.8 \times 10^{-2}$ \\
\hline 64 & $6.1 \times 10^{-11}$ & $2.9 \times 10^{-4}$ & $1.3 \times 10^{-2}$ \\
\hline 128 & $7.0 \times 10^{-21}$ & $3.5 \times 10^{-7}$ & $1.4 \times 10^{-5}$ \\
\hline
\end{tabular}

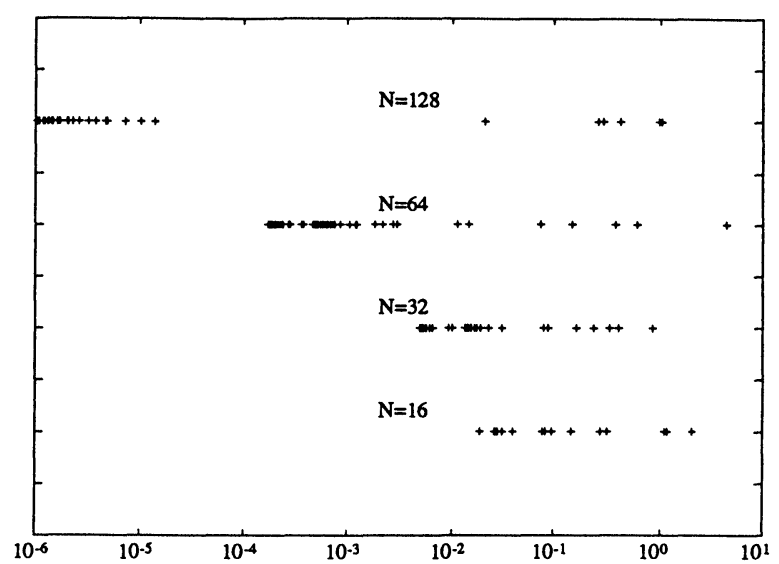

Fig. 1. The eigenvalue distribution of $A^{-1} \triangle A$ for Test Problem 1.

of $\eta$ outliers. A similar phenomenon has been reported in [22] for solving symmetric positive-definite Toeplitz systems with the PCG method.

Test Problem 2. Symmetric $T$ with $\left(\alpha_{T}, \beta_{T}, \gamma_{T}, \delta_{T}\right)=(1,1,1,1)$ and nonsymmetric $T_{H}$ with $\left(\alpha_{H}, \beta_{H}, \gamma_{H}, \delta_{H}\right)=(0,0,1,3)$.

The generating functions of $T$ and $T_{H}$ are chosen to be

$$
T(z)=\frac{0.5+0.3 z^{-1}}{1+0.8 z^{-1}}+\frac{0.5+0.3 z}{1+0.8 z}, \quad H(z)=\frac{0.5-0.4 z}{(1-0.5 z)(1+0.8 z)^{2}}
$$

Note that $B_{T}\left(z^{-1}\right)=1+0.8 z^{-1}$ and $B_{T}(z) D_{T}(z)=(1+0.8 z)^{2}$. Since $B_{T}(z) D_{T}(z)$ and $D_{H}(z)$ have two common roots $\left(\eta_{c}=2\right)$, we know that there are at most three outliers according to (3.11), which is confirmed numerically. The other eigenvalues of $P^{-1} A$ are confined in the disk centered at unity with radius $\epsilon$. Since $\left|h_{-N}\right|>\left|t_{N}\right|=\left|t_{-N}\right|, \epsilon=O\left(\left|h_{-N}\right|\right)$. The clustering radii $\epsilon,\left|t_{N}\right|$ and $\left|h_{-N}\right|$ are listed in Table 2. We apply the CGS method to solve the Toeplitz-plus-Hankel system and plot the convergence history in Fig. 3. The lower and upper four curves are those of the CGS method with and without preconditioning, respectively. The preconditioned CGS method converges faster as $N$ becomes larger, and converges in four iterations asymptotically. In contrast, the CGS method without preconditioning does not converge at all. Although the relation between the convergence rate of the CGS method and the spectrum of the iteration matrix is not clear, our numerical results show that the CGS method converges in a finite number of iterations if the iteration matrix has only a finite number of asymptotic distinct eigenvalues.

Test Problem 3. Nonsymmetric $T$ with $\left(\alpha_{T}, \beta_{T}, \gamma_{T}, \delta_{T}\right)=(1,2,0,0)$ and nonsymmetric $T_{H}$ with $\left(\alpha_{H}, \beta_{H}, \gamma_{H}, \delta_{H}\right)=(1,1,0,0)$. 
TABLE 2

The clustering radius $\epsilon$ of $P^{-1} A$ for Test Problem 2.

\begin{tabular}{|c||c|c|c|}
\hline$N$ & $\left|t_{N}\right|=\left|t_{-N}\right|$ & $\left|h_{-N}\right|$ & $\epsilon$ \\
\hline \hline 16 & $3.5 \times 10^{-3}$ & $2.9 \times 10^{-1}$ & $8.9 \times 10^{-1}$ \\
\hline 32 & $9.9 \times 10^{-5}$ & $1.6 \times 10^{-2}$ & $4.1 \times 10^{-2}$ \\
\hline 64 & $7.8 \times 10^{-8}$ & $2.5 \times 10^{-5}$ & $3.1 \times 10^{-4}$ \\
\hline 128 & $4.9 \times 10^{-14}$ & $3.1 \times 10^{-11}$ & $8.2 \times 10^{-10}$ \\
\hline
\end{tabular}

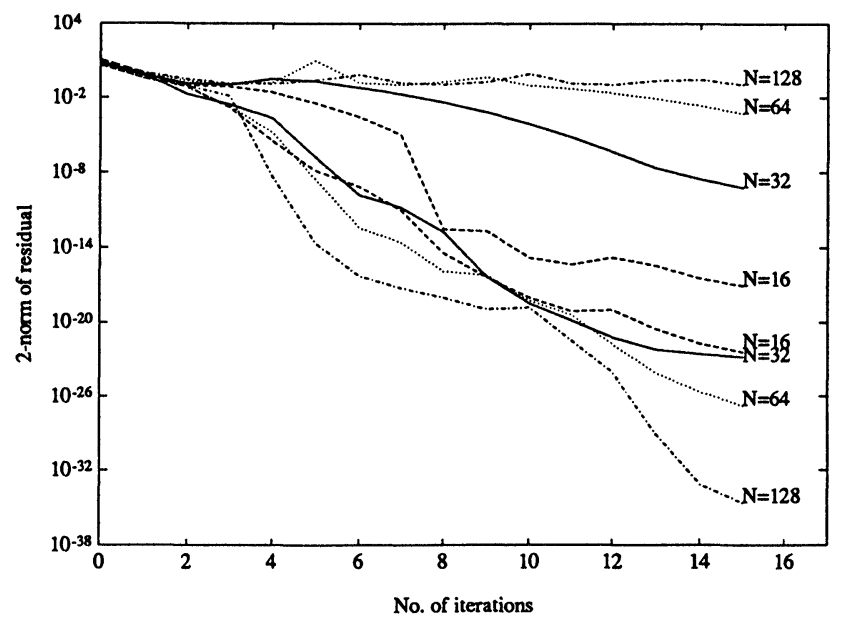

FIG. 2. The convergence history of the PCG method for Test Problem 1.

The generating functions of $T$ and $T_{H}$ are chosen to be

$$
T(z)=\frac{1-0.9 z^{-1}}{\left(1+0.5 z^{-1}\right)\left(1+0.8 z^{-1}\right)}, \quad H(z)=\frac{1+0.5 z^{-1}}{1-0.7 z^{-1}} .
$$

Theorem 2 predicts three outliers, which is confirmed by the experiment for large $N$. All other eigenvalues of $P^{-1} A$ are confined in the disk centered at unity with radius $\epsilon$. The values of $\epsilon,\left|t_{N}\right|$, and $\left|h_{-N}\right|$ are listed in Table 3 . Since $\left|t_{N}\right|>>\left|h_{N}\right|, \epsilon$ decreases at a rate of $O\left(\left|t_{N}\right|\right)$. The convergence history of the CGS method with and without preconditioner is plotted in Fig. 4. They correspond to the lower and upper four curves, respectively. Again, we observe that the CGS method converges in four iterations for large $N$ whereas the CGS method, without preconditioning, does not converge at all.

Test Problem 4. Symmetric nonrational $T$ and $T_{H}$.

The preconditioner $P$ is applied to nonrational Toeplitz-plus-Hankel matrices, where $T$ and $T_{H}$ are symmetric Toeplitz matrices with generating sequences

$$
t_{n}=\frac{1}{|n|+1} \quad \text { and } \quad h_{n}=\frac{1}{(|n|+1)^{1.1}}
$$

respectively. The eigenvalues of $A$ and $P^{-1} A$ are plotted in Fig. 5. Although the spectral properties of $P^{-1} A$ are beyond our analysis in $\S 3$, the preconditioner $P$ still provides a good spectral clustering property. We apply the CGN method to solve the preconditioned system $P^{-1} A \mathbf{x}=P^{-1} \mathbf{b}$, and plot the convergence history of this method with and without preconditioning in Fig. 6, where they correspond to the lower and upper three curves, respectively. It is clear that the CGN method with preconditioner $K$ converges faster than without preconditioning.

Test Problem 5. Ill-conditioned $T$ and $T_{H}$. 


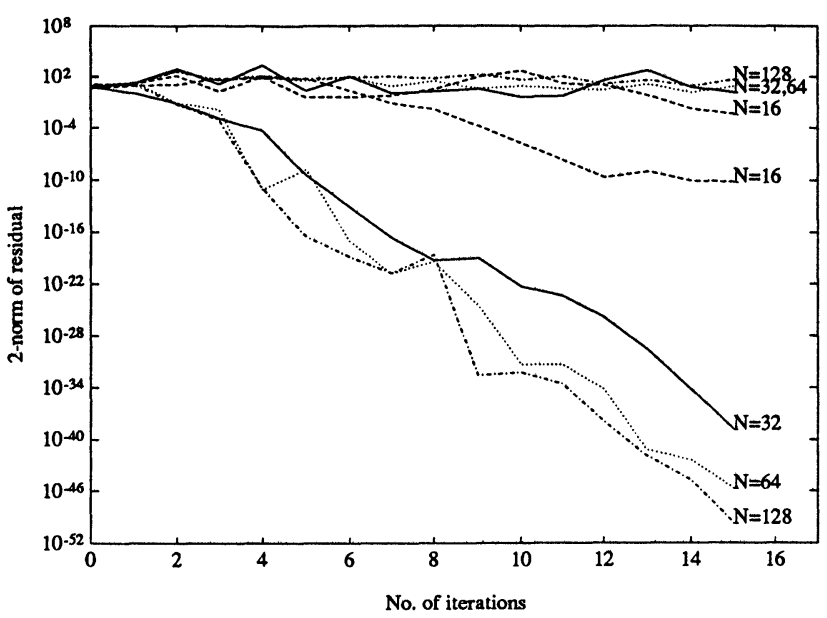

FIG. 3. The convergence history of the CGS method for Test Problem 2.

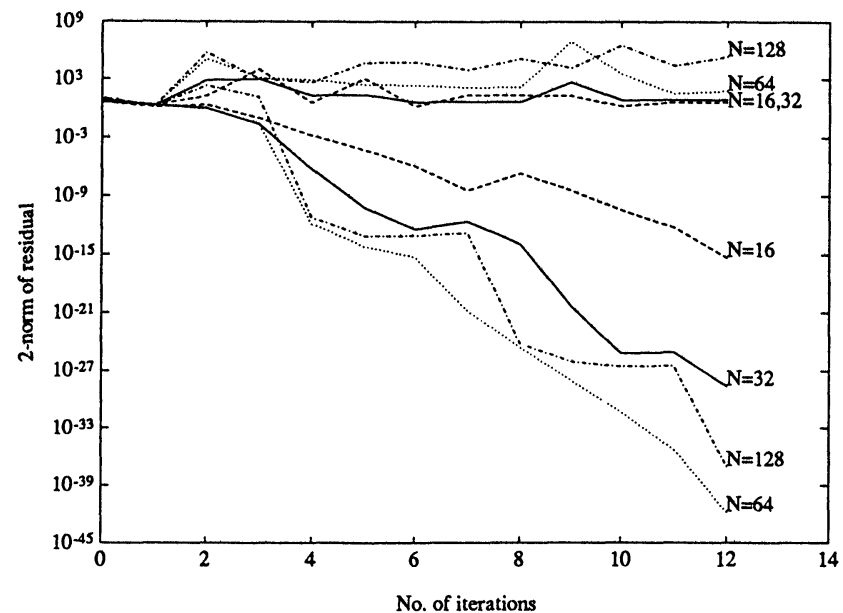

FIG. 4. The convergence history of the CGS method for Test Problem 3.

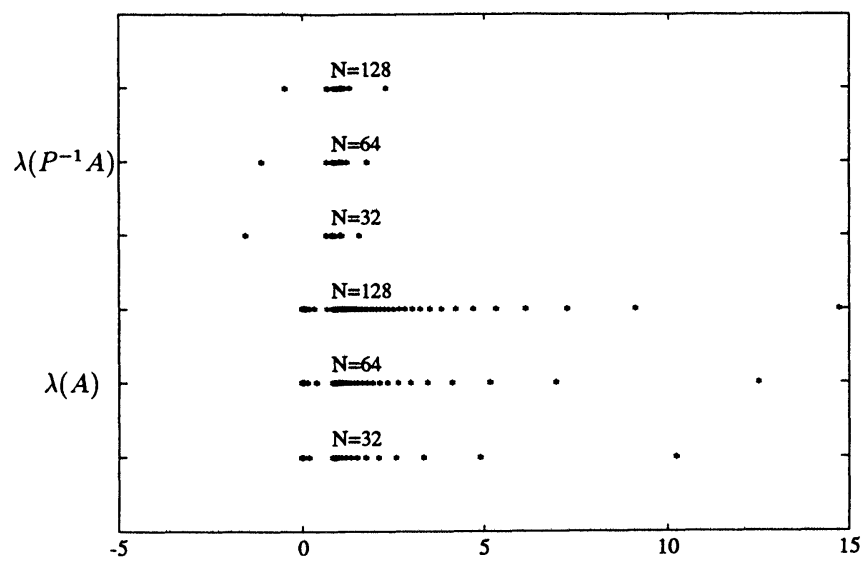

FIG. 5. The eigenvalue distribution of $A$ and $P^{-1} A$ for Test Problem 4. 
TABLE 3

The clustering radius $\epsilon$ of $P^{-1} A$ for Test Problem 3.

\begin{tabular}{|c||c|c|c|}
\hline$N$ & $\left|t_{N}\right|$ & $\left|h_{N}\right|$ & $\epsilon$ \\
\hline \hline 16 & $1.6 \times 10^{-1}$ & $5.7 \times 10^{-3}$ & $1.5 \times 10^{-1}$ \\
\hline 32 & $4.5 \times 10^{-3}$ & $1.9 \times 10^{-5}$ & $1.0 \times 10^{-2}$ \\
\hline 64 & $3.6 \times 10^{-6}$ & $2.1 \times 10^{-10}$ & $8.1 \times 10^{-6}$ \\
\hline 128 & $2.2 \times 10^{-12}$ & $2.6 \times 10^{-20}$ & $1.1 \times 10^{-11}$ \\
\hline
\end{tabular}

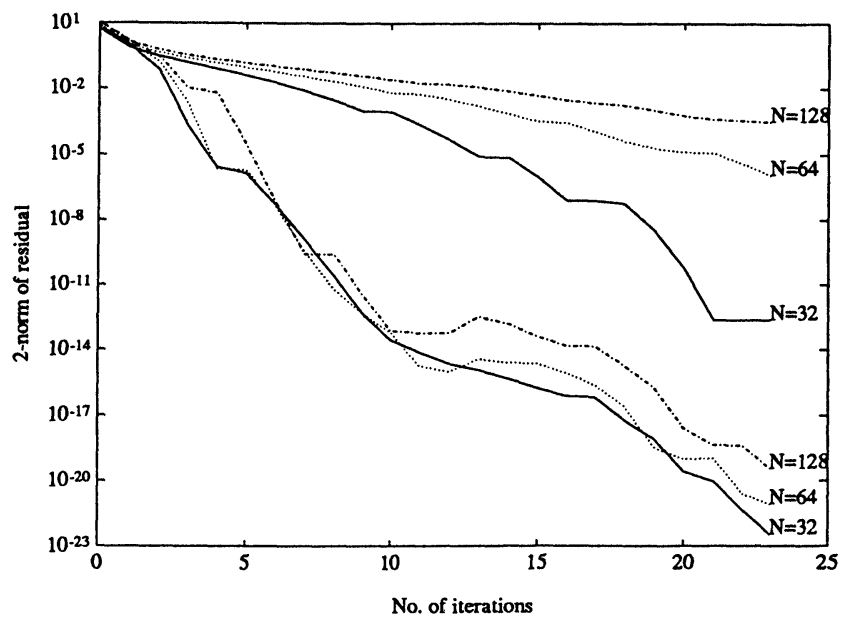

FIG. 6. The convergence history of the CGN method for Test Problem 4.

The generating functions of $T$ and $T_{H}$ are chosen to be

$$
T(z)=\varepsilon_{1}+z^{-1} \quad H(z)=\varepsilon_{2}+z,
$$

where $\varepsilon_{1}$ and $\varepsilon_{2}$ denote some small constants. The $A$ is ill conditioned when both $\varepsilon_{1}$ and $\varepsilon_{2}$ are close to zero. Although $P^{-1} A$ is ill conditioned, it has two distinct eigenvalues, i.e., one outlier close to zero and $(N-1)$ eigenvalues repeated at one. The number of outliers is in fact less than the bound given in Theorem 2. By applying the CGS method to solve the preconditioned system, we observe that the 2-norm of the residual is reduced significantly in two iterations for various values of $\varepsilon_{1}, \varepsilon_{2}$, and $N$.

5. Conclusion. In this research, we generalized the circulant preconditioning technique from Toeplitz to Toeplitz-plus-Hankel matrices. When the Toeplitz and Hankel matrices are both generated by rational functions, we proved that the eigenvalues of the preconditioned matrix are clustered around one, except for a finite number of outliers depending on the order of the generating functions, and that the clustering radius is proportional to the magnitudes of the last elements in Toeplitz and Hankel matrices. With the spectral properties, an $N \times N$ rational Toeplitz-plus-Hankel systems can be solved by preconditioned iterative methods with $O(N \log N)$ operations. Although our discussion focused on real Toeplitz-plus-Hankel systems, the generalization to complex Toeplitz-plus-Hankel systems can be done in a straightforward way.

Acknowledgment. The authors thank the referees for their help in improving the technical presentation of this paper. 


\section{REFERENCES}

[1] Z. S. Agranovich And V. A. Marchenko, The Inverse Problem of Scattering Theory, Gordon and Breach, New York, 1963.

[2] J. G. BerRyman AND R. R. GREene, Discrete inverse methods for elastic waves in layered media, Geophysics, 45 (1980), pp. 213-233.

[3] A. M. BRUCKSTEIN AND T. KAILATH, Inverse scattering for discrete transmission-line models, SIAM Rev., 29 (1987), pp. 359-389.

[4] A. M. Bruckstein, T. Kailath, I. Koltracht, and P. Lancaster, On the reconstruction of layered media from reflection data, SIAM J. Matrix Anal. Appl., 12 (1991), pp. 24-40.

[5] J. R. BUNCH, Stability of methods for solving Toeplitz systems of equations, SIAM J. Sci. Statist. Comput., 6 (1985), pp. 349-364.

[6] K. ChadAN AND P. C. Sabatier, Inverse problems in quantum scattering theory, SpringerVerlag, New York, 1977.

[7] R. H. Chan, Circulant preconditioners for Hermitian Toeplitz system, SIAM J. Matrix Anal. Appl., 10 (1989), pp. 542-550.

[8] R. H. ChAN AND G. STRANG, Toeplitz equations by conjugate gradients with circulant preconditioner, SIAM J. Sci. Statist. Comput., 10 (1989), pp. 104-119.

[9] T. F. ChAN, An optimal circulant preconditioner for Toeplitz systems, SIAM J. Sci. Statist. Comput., 9 (1988), pp. 766-771.

[10] G. CYвENKo, The numerical stability of the Levinson-Durbin algorithm for Toeplitz systems of equations, SIAM J. Sci. Statist. Comput., 1 (1980), pp. 303-319.

[11] P. Delsarte AND Y. V. Genin, The split Levinson algorithm, IEEE Trans. Acoust. Speech Signal Process., ASSP-34 (1986), pp. 470-478.

[12] P. DEWILDE AND H. DYM, Schur recursions, error formulas and convergence of rational estimators for stationary stochastic processes, IEEE Trans. Information Theory, IT-27 (1981), pp. 446-461.

[13] P. Dewilde, A. C. Vieira, AND T. KAILATH, On a generalized Szegö-Levinson realization algorithm for optimal linear predictors based on a network synthesis approach, IEEE Trans. Circuits and Systems, CAS-25 (1978), pp. 663-675.

[14] I. M. GELFAND AND B. M. LEVITAN, On the determination of a differential equation from its spectral function, Amer. Math. Soc. Trans., 1 (1955), pp. 253-304.

[15] G. H. Golub ANd C. F. VAN Loan, Matrix Computations, John Hopkins University Press, Baltimore, MD, 1983.

[16] G. HeINIG AND K. Rost, On the inverse of Toeplitz-plus-Hankel matrices, Linear Algebra Appl., 106 (1988), pp. 39-52.

[17] — Matrix representation of Toeplitz-plus-Hankel matrix inverses, Linear Algebra Appl., 113 (1989), pp. 65-78.

[18] M. R. HeStenes AND E. STIEFEL, Methods of conjugate gradients for solving linear systems, J. Res. Nat. Bur. Standards, 49 (1952), pp. 409-436.

[19] T. HUCKLE, Circulant and skew-circulant matrices for solving Toeplitz matrices problems, SIAM J. Matrix Anal. Appl., 13 (1992), pp. 767-777.

[20] M. G. KREIN, On a method for the effective solution of the inverse boundary value problem, Dokl. Akad. Nauk. SSSR, 94 (1954), pp. 987-990. (In Russian.)

[21] H. KRISHNA AND S. MORGERA, The Levinson recurrence and fast algorithm for solving Toeplitz system of linear equations, IEEE Trans. Acoust. Speech Signal Process., ASSP-35 (1987), pp. 839-848.

[22] T. K. KU AND C. J. KUo, Design and analysis of Toeplitz preconditioners, IEEE Trans. Acoust. Speech Signal Process., 40 (1992), pp. 129-141.

[23] — A minimum-phase LU factorization preconditioner for Toeplitz matrices, SIAM J. Sci. Statist. Comput., 13 (1992), pp. 1470-1487.

[24] - Spectral properties of preconditioned rational Toeplitz matrices, SIAM J. Matrix Anal. Appl., 14 (1993), to appear.

[25] — Spectral properties of preconditioned rational Toeplitz matrices: The nonsymmetric case, SIAM J. Matrix Anal. Appl., 14 (1993), to appear.

[26] J. M. MENDEL AND F. HABIBI-Ashrafi, A survey of approaches to solving inverse problems for lossless layered media systems, IEEE Trans. Geosci. Remote Sensing, GE-18 (1980), pp. 320-330.

[27] G. A. Merchant AND T. W. Parks, Efficient solution of a Toeplitz-plus-Hankel coefficient matrix system of equations, IEEE Trans. Acoust. Speech Signal Process., ASSP-30 (1982), pp. 40-44.

[28] Y. SAAD AND M. H. SchUltz, GMRES: A generalized minimum residual algorithm for solving 
nonsymmetric linear systems, SIAM J. Sci. Statist. Comput., 7 (1986), pp. 856-869.

[29] P. SonNEVELD, CGS, a fast Lanczos-type solver for nonsymmetric linear systems, SIAM J. Sci. Statist. Comput., 10 (1989), pp. 36-52.

[30] G. Strang, A proposal for Toeplitz matrix calculations, Stud. Appl. Math., 74 (1986), pp. 171176.

[31] L. N. Trefethen, Approximation theory and numerical linear algebra, in Algorithms for Approximation II, M. Cox and J. C. Mason, eds., Chapman, London, 1988.

[32] E. TYRTYSHNIKOV, Optimal and super-optimal circulant preconditioners, SIAM J. Matrix Anal. Appl., 13 (1992), pp. 459-473.

[33] J. H. Wilkinson, The Algebraic Eigenvalue Problem, Oxford University Press, London, 1965. 